\title{
Should Acid Suppressive Therapy Improve Chronic Cough?
}

\author{
(Chest 2013;143:605-612)
}

Jung Hwan Oh

Division of Gastroenterology, Department of Internal Medicine, The Catholic University of Korea College of Medicine, Seoul, Korea

\section{Summary}

Gastroesophageal reflux disease (GERD) may cause laryngopharyngeal reflux, which results in mucosal exposure of the pharynx, larynx, or pulmonary system to the different components of the gastric refluxate, and so provokes symptoms including throat discomfort, hoarseness, globus pharyngeus and chronic cough. Chronic cough, defined as a cough lasting more than 8 weeks, is a common problem in primary care. The most common etiology of chronic cough in adults is upper airway cough syndrome secondary to postnasal drip, asthma, or GERD. ${ }^{1}$

Kahrilas et $\mathrm{al}^{2}$ has recently reported a systemic review regarding response of chronic cough to acid suppressive therapy in patients with GERD. ${ }^{2}$ A systemic search of Pubmed and Embase was conducted, which included placebo-controlled clinical trials reporting data on the effect of acid suppressive therapy on chronic cough in patients with GERD or laryngopharyngeal reflux by objective measures and/or reflux symptoms. Finally, they reviewed nine randomized controlled trials that treated patients with acid suppression. Among them, 8 trials used proton pump inhibitors (PPIs; daily or bid for 8-16 weeks), while 1 trial used ranitidine. Therapeutic gain was greater in patients with pathologic esophageal acid exposure (mean 21.5\%; range, 12.5-35.8\%) than in those without pathologic esophageal acid exposure (range, 0.0-8.6\%). Patients with heartburn were excluded from the 2 studies which included patients without acid exposure. The mean placebo response rate was $13.8 \%$. Although study design and subjective outcomes were variable in each study, the authors concluded that a therapeutic benefit for acid suppressive therapy in patients with chronic cough cannot be dismissed. It would be much more effective in selective patients who are favorable to therapy based on symptoms or reflux testing.

\section{Comment}

There is paucity of evidence that acid suppressive therapy would improve reflux-associated cough. There are several difficulties, which are small patient's number, variety of study design and cough outcome measures, in interpreting whether it would be appropriate. In addition, there is no gold standard for establishing the association between GERD and atypical symptoms of GERD because many of them may have a variety of etiologies. In patients with chronic cough who have prominent upper gastrointestinal symptoms consistent with GERD, an empiric trial of medical acid suppressive therapy is recommended according to ACCP guideline. ${ }^{3}$ In Cochrane database review, they analyzed nine adult studies comparing PPI (2 to 3 months) to placebo for

Received: June 10, 2013 Revised: June 21, 2013 Accepted: June 22, 2013

(c) This is an Open Access article distributed under the terms of the Creative Commons Attribution Non-Commercial License (http://creativecommons. org/licenses/by-nc/3.0) which permits unrestricted non-commercial use, distribution, and reproduction in any medium, provided the original work is properly cited.

*Correspondence: Jung Hwan Oh, MD

Department of Internal Medicine, The Catholic University of Korea College of Medicine, St. Paul's Hospital, 180, Wangsan-ro, Dongdaemun-gu, Seoul 130-709, Korea

Financial support: None.

Tel: +82-2-958-2343, Fax: +82-2-968-7250, E-mail: ojh@catholic.ac.kr

Conflicts of interest: None. 
various outcomes in the meta-analysis. However, there was insufficient evidence to conclude definitely that GERD treatment with PPI is universally beneficial for cough associated with GERD. ${ }^{4}$ Recent 2 studies which were conducted in double-blind, placebo-controlled manner supported that PPI did not improve cough symptoms. Esomeprazole of $40 \mathrm{mg}$ twice daily for 12 weeks was not effective for patients with chronic cough. However, they did not include patients who had heartburn symptoms greater than 2 times per month. ${ }^{5}$ Another study showed that esomeprazole $20 \mathrm{mg}$ twice daily for 8 weeks did not lead to a significant clinical effect. ${ }^{6}$

Kahrilas et $\mathrm{al}^{2}$ tried to compare the response of chronic cough to acid suppressive therapy between 2 groups based on with and without objective measures of GERD. ${ }^{2}$ Acid suppressive therapy was much more effective in patients with pathologic esophageal acid exposure than in those without acid exposure. They, however, pointed out that a therapeutic benefit of acid suppression, even in patients with pathologic esophageal acid exposure, seemed not to be satisfactory. First, hypersensitivity of the cough reflux would explain the poorer response than expected. Smith et $\mathrm{al}^{7}$ suggested reflux could temporally associate with cough irrespective of possible underlying cause. That is, in addition to reflux potentially inducing cough, cough could also provoke reflux by increasing transient lower esophageal sphincter relaxations. Increased cough reflex sensitivity may be facilitating reflux-induced cough and cough-induced reflux. Hence, it may be possible to have a self-perpetuating cycle in some patients with chronic cough. These results may partly explain why many patients with chronic cough are comparatively refractory to PPI therapy compared to patients with typical reflux symptoms, such as heartburn and regurgitation. Second, acid reflux induced by coughing may cause many patients with chronic cough to have pathologic esophageal acid exposure. Third, there is potential for weekly acidic reflux resulting from acid suppressive therapy to provoke perpetuating cough.

To diminish the failure of acid suppressive therapy, the strict patient selection should be taken into account. Recent study pointed out the importance of history taking in the assessment of patients since the symptoms were indicative of the underlying cause of cough. ${ }^{8}$ In other words, acid taste and heartburn symptom were positive markers predicting good response. However, $43 \%$ of patients with chronic cough did not have GERD symptoms. ${ }^{9}$ Therefore, dyspeptic symptoms are frequently absent in spite of reflux related cough. The presence of having pathologic acid exposure by reflux symptoms and/or objective meas- ures may be a valuable sign to predict the response to acid suppressive therapy in patients with chronic cough. Lack of response to empirical therapy should not be followed by increasing treatment duration and dosage in real clinical field. Instead, other causes such as allergy, sinus and pulmonary disease should be searched. ${ }^{10}$ Kahrilas et al $^{2}$ also suggested some guideline for future clinical trials. Crossover design should be avoided. Cough outcome measure and objective evidence of reflux should be verified. Acoustic cough-monitoring device would be an ideal diagnostic test which may more precisely predict the responsiveness to acid suppressive therapy in patients with chronic cough. With regard to trial duration, 8 weeks would be optimal, however, it was contended a longer acid suppression for at least 4 months should be warranted in patients with chronic cough. ${ }^{11}$ Based on Asia-Pacific consensus on the management of GERD, patients with chronic cough and typical GERD symptoms should be offered twice daily PPI therapy after exclusion of non-GERD etiologies. $^{12}$

Although this study describes that the effectiveness of acid suppressive therapy could be limited in patients with chronic cough even in whom with pathologic esophageal acid exposure, other causes should be considered when the response outcome is disappointing after 2 to 4 months of PPI therapy.

\section{References}

1. Kwon NH, Oh MJ, Min TH, Lee BJ, Choi DC. Causes and clinical features of subacute cough. Chest 2006;129:1142-1147.

2. Kahrilas PJ, Howden CW, Hughes N, Molloy-Bland M. Response of chronic cough to acid-suppressive therapy in patients with gastroesophageal reflux disease. Chest 2013;143:605-612.

3. Irwin RS. Chronic cough due to gastroesophageal reflux disease: ACCP evidence-based clinical practice guidelines. Chest 2006;129: 80S-94S.

4. Chang AB, Lasserson TJ, Gaffney J, Connor FL, Garske LA. Gastro-oesophageal reflux treatment for prolonged non-specific cough in children and adults. Cochrane Database Syst Rev 2011; (1):CD004823

5. Shaheen NJ, Crockett SD, Bright SD, et al. Randomised clinical trial: high-dose acid suppression for chronic cough - a double-blind, placebo-controlled study. Aliment Pharmacol Ther 2011;33:225234.

6. Faruqi S, Molyneux ID, Fathi H, Wright C, Thompson R, Morice AH. Chronic cough and esomeprazole: a double-blind placebo-controlled parallel study. Respirology 2011;16:1150-1156.

7. Smith JA, Decalmer S, Kelsall A, et al. Acoustic cough-reflux associations in chronic cough: potential triggers and mechanisms. Gastroenterology 2010;139:754-762.

8. Ojoo JC, Everett CF, Mulrennan SA, Faruqi S, Kastelik JA, Morice AH. Management of patients with chronic cough using a 
clinical protocol: a prospective observational study. Cough 2013;9:2.

9. Poe RH, Kallay MC. Chronic cough and gastroesophageal reflux disease: experience with specific therapy for diagnosis and treatment. Chest 2003;123:679-684.

10. Vaezi MF. Chronic cough and gastroesophageal reflux disease: how do we establish a causal link? Chest 2013;143:587-589.
11. Spechler SJ, Lee E, Ahnen D, et al. Long-term outcome of medical and surgical therapies for gastroesophageal reflux disease: follow-up of a randomized controlled trial. JAMA 2001;285:2331-2338.

12. Fock KM, Talley NJ, Fass R, et al. Asia-Pacific consensus on the management of gastroesophageal reflux disease: update. J Gastroenterol Hepatol 2008;23:8-22. 